\title{
Towards a new concept for high sensitivity Compton scatter emission imaging
}

\author{
Mai K. Nguyen \\ mai.nguyen-verger@u-cergy.fr \\ Clémence Driol \\ clemence.driol@u-cergy.fr \\ Tuong T. Truong \\ truong@u-cergy.fr
}

Habib Zaidi

habib.zaidi@hcuge.ch

\author{
ETIS / CNRS UMR 8051 / ENSEA / Université de Cergy-Pontoise, 6 avenue du Ponceau, 95014 Cergy- \\ Pontoise Cedex, France \\ LPTM / CNRS UMR 8089 / Université de Cergy-Pontoise, 2 rue Adolphe Chauvin, 95302 Cergy- \\ Pontoise Cedex, France \\ LPTM / CNRS UMR 8089 / Université de Cergy-Pontoise, 2 rue Adolphe Chauvin, 95302 Cergy- \\ Pontoise Cedex, France \\ Geneva University Hospital, Division of Nuclear Medicine, CH-1211 Geneva 4, Switzerland
}

A new efficient scheme for imaging gamma-emitting objects is advocated in this work. It is elaborated on the recent idea of collecting data, using a detector equipped with a parallel-hole collimator, from Compton scattered photons to reconstruct an object in three-dimensions. This paper examines a working mode without collimation, which should increase its sensitivity and field of view. To simplify the otherwise complex mathematical formulation, we choose to discuss the image formation process in two-dimensions, which can be implemented with a slit collimator. Comparison with the standard collimated case, via the analysis of the shapes of the respective point spread functions (PSF), shows marked improvements and numerical simulation results, obtained using a brain phantom, support the viability and attractiveness of this new imaging modality. [DOI: 10.2971/jeos.2008.08010]

Keywords: Emission imaging, sensitivity, Compton scattering, image formation, image reconstruction

\section{INTRODUCTION}

Emission imaging using gamma-emitting sources is widely used in numerous fields such as medical imaging, gamma astronomy, non-destructive testing and environmental survey. In conventional nuclear imaging, a collimated gamma camera rotates in space to collect primary radiation emitted by the object (human organ) under investigation. In this case, Compton scattered radiation is generally considered as noise hindering image quality and quantitative accuracy and consequently appropriate scatter correction procedures should be applied [1].

Recently an interesting novel imaging concept, which precisely uses, as imaging agent, radiation scattered by the object (instead of primary radiation), has been proposed. A spatially fixed collimated gamma camera records now projection data labeled by the energy of scattered radiation (or equivalently its scattering angle). It has then been shown that the reconstruction of a three-dimensional object is feasible using this data acquisition geometry [2]-[5]. However in this situation, the image sensitivity is considerably affected owing to the presence of the parallel-hole collimator. Only about one out of $10^{4}$ scattered photons reaches the detector [6]. Therefore in order to record a much larger amount of scattered radiation, we propose to extend the working principle of this Compton imaging concept to a functioning modality without collimator, as depicted in Figure 1. It can clearly be seen that this concept differs from earlier proposals on scattered radiation imaging [7]-[10], in particular:
- Compton tomography [11], which reconstructs the electron density of the object (instead of its activity density), and uses a moving point-like detector collecting scattered radiation from an external radiation source,

- Compton camera [12]-[14], which reconstructs the activity density of an object from scattered radiation using coincidence measurements between a site on a solid-state scatter layer-detector and another site on a scintillation crystal-based absorption-detector.

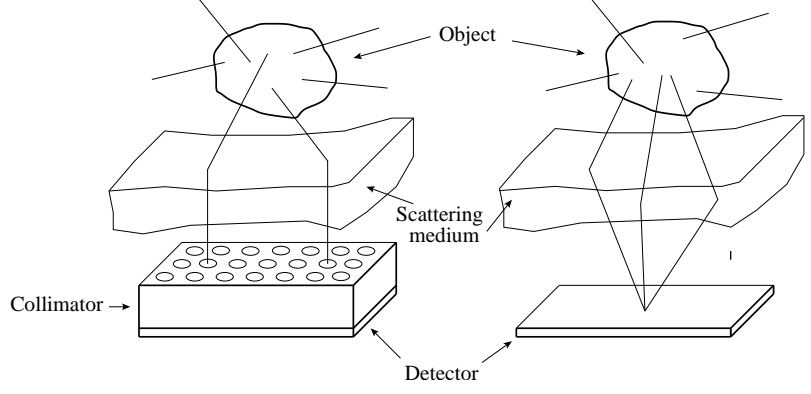

FIG. 1 Illustration of collimated and uncollimated scattered radiation emission imaging.

The quantity to be reconstructed in emission imaging is the spatial distribution of the radio-tracer in the patient's body. As the true three-dimensional problem involves an open detection geometry for scattered photons thus requiring a complex mathematical formulation, we shall first study its twodimensional counterpart to test the viability of this idea. To 
this end, we give a careful analysis of the image formation process for a two-dimensional geometry achieved using a slit collimator. The corresponding PSF is derived and will be compared with the previous case where a parallel-hole collimator is present. Next we perform numerical reconstruction of the activity distribution of the Shepp-Logan phantom obtained from simulated scattered radiation collected using the above mentioned detection geometry to illustrate the working principle of this concept. The current concept might be useful for the solid-state based SPECT imager proposed recently in Ref. [15] which by appropriate consideration of the trade-off of the system sensitivity and spatial resolution offers many advantages compared to conventional scintillation camera-based designs.

\section{IMAGE FORMATION}

To understand the image formation process when detecting scattered radiation, we follow radiation propagation in a two-dimensional (2D) slice (or thin section) of a scattering medium with an electronic density $n_{e}$ assumed to be approximately constant. This can in principle be implemented using a slit collimator placed directly above the scintillation detector (Figure 2). We also consider that most scattered photons will undergo single Compton scattering with electrons inside the object given that higher order scattered photons have much lower probability of being detected in the defined pulse-height energy window, as reported in many Monte Carlo simulation studies [16]. Moreover, to concentrate mainly on Compton scattered radiation, the attenuation inherent to this imaging modality will be left out as working hypothesis. It should be noted that the photoelectric absorption cross-section for the energies of interest in nuclear medicine is very low.

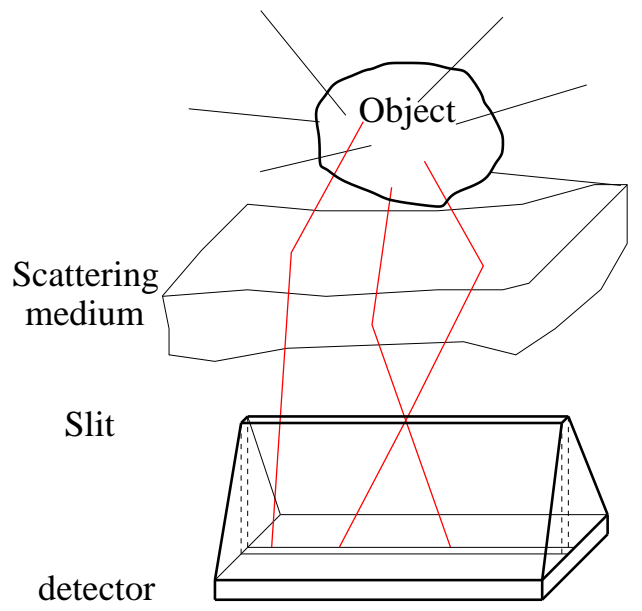

FIG. 2 Two-dimensional detection geometry using a dedicated slit collimator.

Let

- $\mathbf{S}=\left(x_{S}, y_{S}\right)$ be a gamma-emitting point source of an object described by its activity density $f(\mathbf{S})$ (number of photons emitted per unit time isotropically in all directions,
- $\mathbf{M}=\left(x_{M}, y_{M}\right)$, a scattering site in the medium,

- $n_{e}(\mathbf{M})$, the electron density at site $\mathbf{M}$,

- $\mathbf{D}=\left(x_{D}, y_{D}\right)$, a detection site on a linear detector which collects the photon flux density at a photon energy $E_{\omega}$ (see Eq. (1)).

The energy of the scattered photon is related to the scattering angle $\omega$ by the Compton equation:

$$
E_{\omega}=E_{0} \frac{1}{1+\varepsilon(1-\cos \omega)},
$$

where $E_{0}$ is the emitted photon energy, $\varepsilon=E_{0} / m c^{2}$ and $m c^{2}$ the rest energy of the electron.

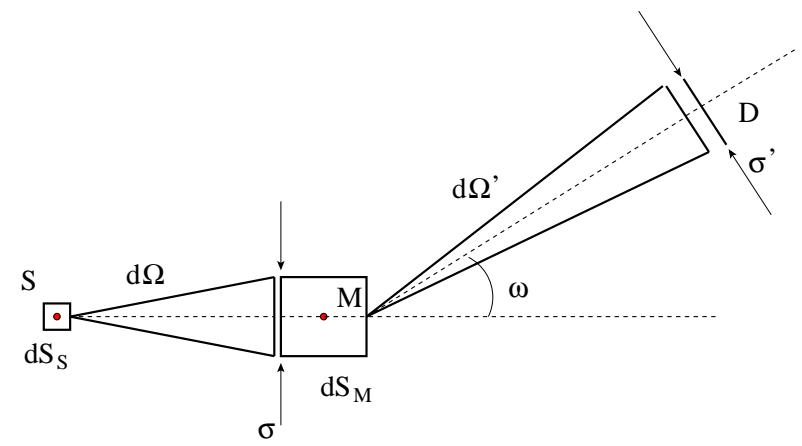

FIG. 3 Geometry of Compton scattering showing source site $\mathbf{S}$, scattering site $\mathbf{M}$ and detection site $\mathbf{D}$ of width $\sigma^{\prime}$.

From Figure 3, one can see that the photon flux density reaching a site $\mathbf{M}$ is the number of photons emitted into the angular fan $d \Omega$ per unit length and per unit time:

$$
\frac{f(\mathbf{S}) d \mathbf{S}_{S}}{2 \pi} 2 \arctan \left(\frac{\sigma}{2|S M|}\right) \frac{1}{\sigma^{\prime}}
$$

where $|S M|$ is the distance between sites $\mathbf{S}$ and $\mathbf{M}$ and $d \mathbf{S}_{S}$ the area element around $\mathbf{S}$. The fraction of photons scattered in the direction making an angle $\omega$ with the incident direction depends on the Compton differential cross section $\sigma_{S}^{C}(\omega)$ (which has the dimension of length in 2D) and on the number of electrons $n_{e}(\mathbf{M}) d \mathbf{S}_{M}$ at site $\mathbf{M}, d \mathbf{S}_{M}$ being the integration area element around $\mathbf{M}$. Hence the scattering photon flux density received at the detection site $\mathbf{D}$ is given by :

$$
\begin{aligned}
& d \phi(\mathbf{D}, \omega \mid \mathbf{S})=\frac{f(\mathbf{S}) d \mathbf{S}_{S}}{2 \pi} 2 \arctan \left(\frac{\sigma}{2|S M|}\right) \frac{1}{\sigma} \\
& \times n_{e}(\mathbf{M}) d \mathbf{S}_{M} \sigma_{S}^{C}(\omega) 2 \arctan \left(\frac{\sigma^{\prime}}{2|M D|}\right) \frac{1}{\sigma^{\prime}} \cos \theta,
\end{aligned}
$$

where $\theta$ is the angle between the outgoing photon unit vector with the detector normal unit vector, $|M D|$ the distance from scattering site $\mathbf{M}$ to detection site $\mathbf{D}$. In fact, for a given point source $\mathbf{S}$, there will be two scattering sites $\mathbf{M}_{1}$ and $\mathbf{M}_{2}$ located on two arcs of a circle subtending an angle $(\pi-\omega)$, as shown in Figure 4. 
The total photon flux density at a site $\mathbf{D}$ is $g(\mathbf{D}, \omega)$, the integral over all source sites and all scattering sites such that the scattering angle is $\omega$. This last constraint is expressed by a $\delta$ function in the integration as

$$
g(\mathbf{D}, \omega)=\iint d \phi(\mathbf{D}, \omega \mid \mathbf{S}) \delta(\widehat{S M D}-(\pi-\omega)),
$$

where $\widehat{S M D}$ is the angle at vertex $\mathbf{M}$ of the triangle $S M D$ (see Figure 4). Eq. (4) describes the basic image formation process from scattered radiation measured on the detection system shown above.

\section{COMPUTATION OF THE SYSTEM POINT SPREAD FUNCTION}

We now study and compute the essential component of this new imaging concept, the point spread function (PSF). It is, by definition, the response of the imaging system to a single point source at site S. Eq. (4) can be rewritten in terms of the PSF as follows:

$$
g(\mathbf{D}, \omega)=\int d \mathbf{S}_{S} f(\mathbf{S}) \operatorname{PSF}(\mathbf{D}, \omega \mid \mathbf{S})
$$

As shown earlier for a detection geometry corresponding to Figure 2, the scattering sites due to a single point source $\mathbf{S}$ are located on two circular arcs subtending an angle $(\pi-\omega)$ (Eq. (4)). The photon flux density received at site $\mathbf{D}$ is then given by an integration over these two circular arcs (see Figure 4).

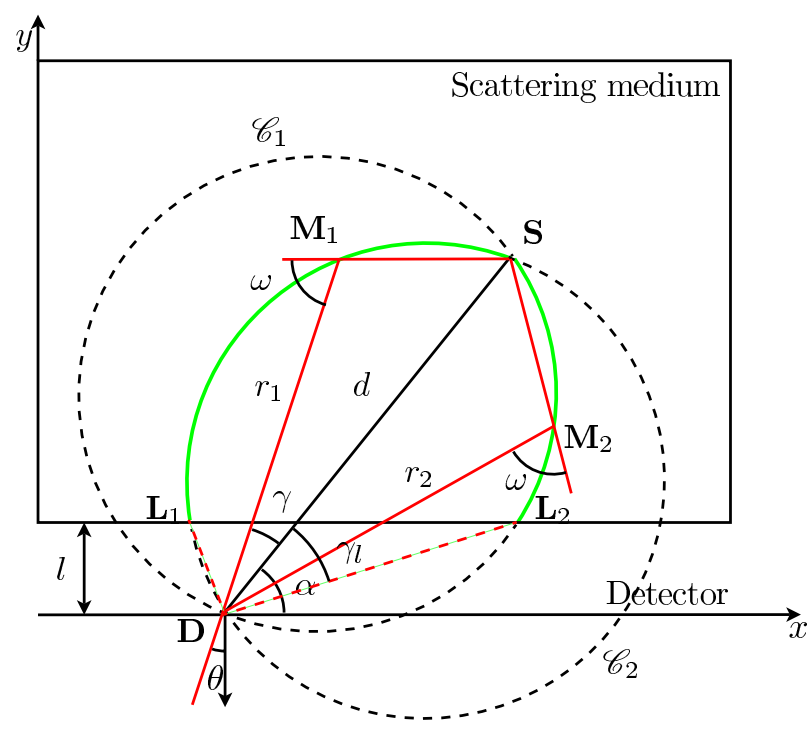

FIG. 4 Locus of scattering sites due to a single point source.

For computation ease, we shall assume a uniform electron density $n_{e}$ and use polar coordinates $(r, \gamma)$, such that $\mathbf{S}=$ $(d, \alpha)$ with $D S=d$ and $\mathbf{M}=(r, \gamma)$, with $D M=r$ and $\overrightarrow{D M} \cdot \overrightarrow{D S}=r d \cos \gamma$.

The circular arcs have polar equations:

$$
r=d \frac{\sin (\omega-\gamma)}{\sin \omega} \quad \text { and } \quad r=d \frac{\sin (\omega+\gamma)}{\sin \omega}
$$

where $\gamma$ is the angle between $\overrightarrow{D S}$ and $\overrightarrow{D M}$. The distance $|S M|$ can be extracted from a simple identity in the triangle DSM:

$$
|S M|=d \frac{\sin \gamma}{\sin \omega}
$$

And the integration area $d \mathbf{S}_{M}$ is now reduced to the arc element:

$$
\sqrt{d r^{2}+r^{2} d \gamma^{2}}=\frac{d}{\sin \omega} d \gamma
$$

Hence the PSF is given by the sum of the two integrations on $\gamma$ :

$$
\begin{gathered}
\operatorname{PSF}(\mathbf{D}, \omega \mid \mathbf{S})=\frac{K(\omega) d}{\sigma \sigma^{\prime} \sin \omega} \sum_{2} \int_{\operatorname{ArcS}}^{\gamma_{l}(\omega)} d \gamma \cos \theta(\gamma) \\
\arctan \left(\frac{\sigma}{2 d} \frac{\sin \omega}{\sin \gamma}\right) \arctan \left(\frac{\sigma^{\prime}}{2 d} \frac{\sin \omega}{\sin (\omega \pm \gamma)}\right),
\end{gathered}
$$

where $K(\omega)=4 n_{e} \sigma_{S}^{C}(\omega) f_{0} / 2 \pi, \sigma_{S}^{C}(\omega)$ the differential Compton cross-section at scattering angle $\omega, f_{0}$ the intensity of the single point source and $\cos \theta=\sin (\alpha-\gamma)$ if the detector lies along the $x$-axis and $l$ is the distance between the line detector and the linear lower boundary of the medium $L$. The integration is carried out over the points inside the scattering medium. Therefore when the medium is of finite extent, the limit of the integration $\gamma_{l}(\omega)$, which corresponds to the intersection of the arcs of circle with the scattering medium, should be calculated beforehand, (see Figure 4).

Now if the parallel-hole collimator is mounted on the detector, then only one scattering site $\mathbf{M}$, located on the perpendicular to the detector at site $\mathbf{D}$, will contribute to detection site D, (see Figure 5). Thus the integration on $\gamma$ is restricted by a delta function (neglecting the detector response function) which picks out only the corresponding value of $\gamma$, i.e.:

$$
\gamma_{c o l}=\frac{\pi}{2}-\alpha
$$

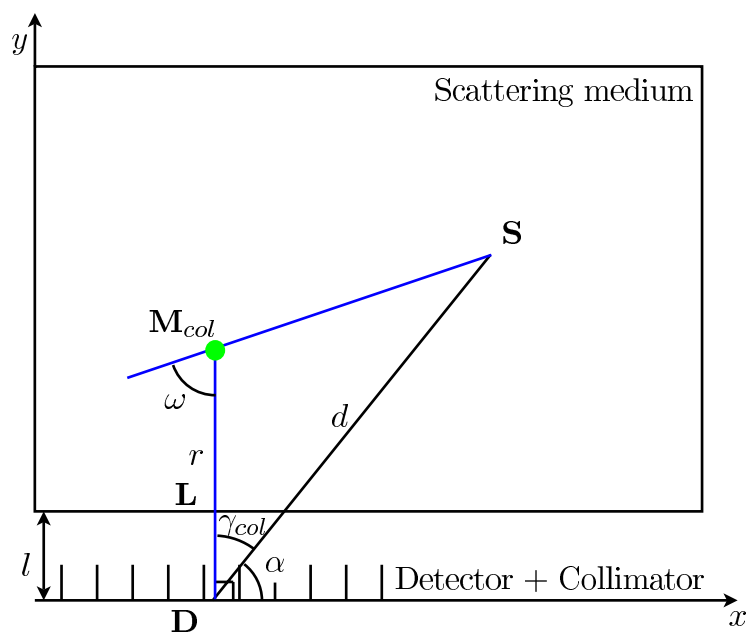

FIG. 5 Scattering site in the presence of a parallel-hole collimator from a single point source. 
The resulting PSF expression for a parallel-hole collimated detector is:

$$
\begin{aligned}
& \operatorname{PSF}_{c o l}(\mathbf{D}, \omega \mid \mathbf{S})=\frac{K(\omega) d}{\sigma \sigma^{\prime} \sin \omega} \\
& \arctan \left(\frac{\sigma}{2 d} \frac{\sin \omega}{\cos \alpha}\right) \arctan \left(\frac{\sigma^{\prime}}{2 d} \frac{\sin \omega}{ \pm \cos (\alpha \mp \omega)}\right) .
\end{aligned}
$$

Now at a fixed scattering angle $\omega$, the PSF curve as function of the detector position with parallel collimator has a Mexican hat shape (Figure 6), whereas the PSF curve without collimator has a wide Lorentzian shape (see Figure 7). In addition the amplitude of the PSF with the slit collimator is substantially higher.

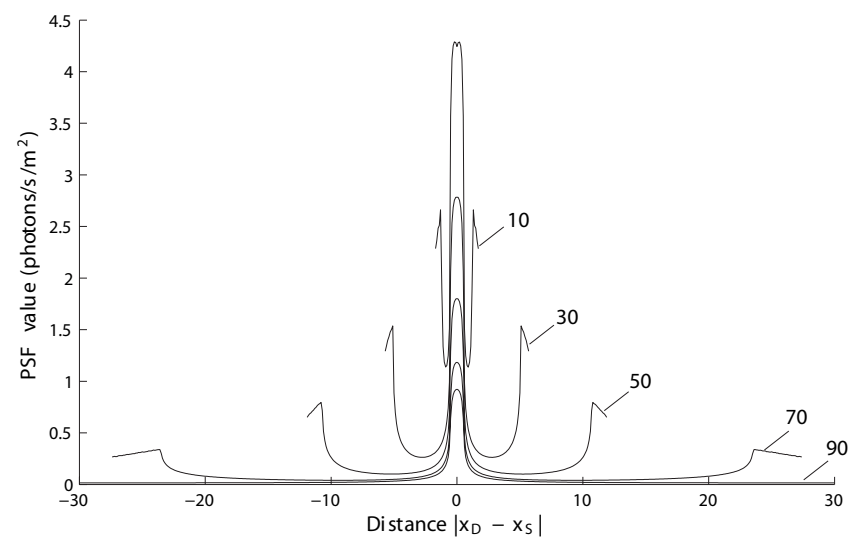

FIG. 6 Shapes of PSFs corresponding to a collimated detection geometry.

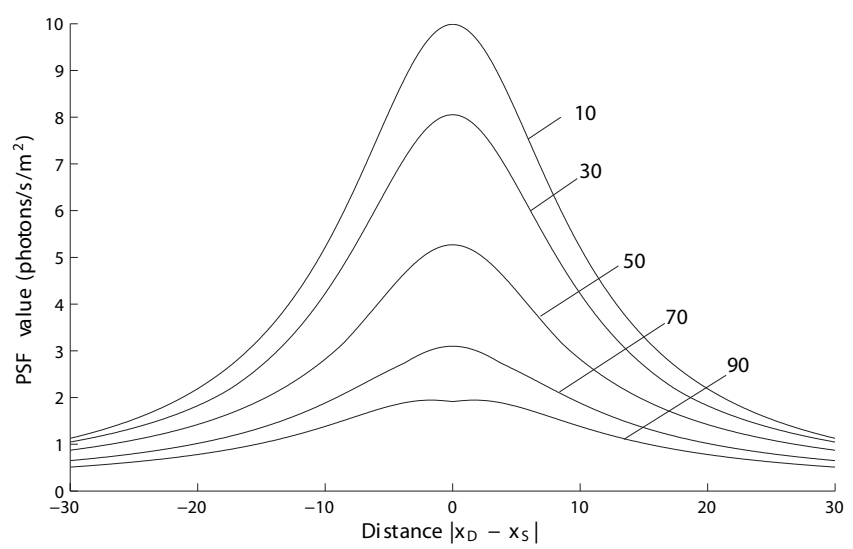

FIG. 7 Shapes of PSFs corresponding to a detection geometry equipped with a slit collimator.

For example at a scattering angle of 30 degrees, Figure 8 shows that the PSF with the slit collimator is about 10 times stronger than the PSF with the parallel collimator.

\section{NUMERICAL RECONSTRUCTION RESULTS}

As an illustration of the potential of this new imaging concept, we carried out numerical simulations using the twodimensional brain phantom. Simulation data generated using

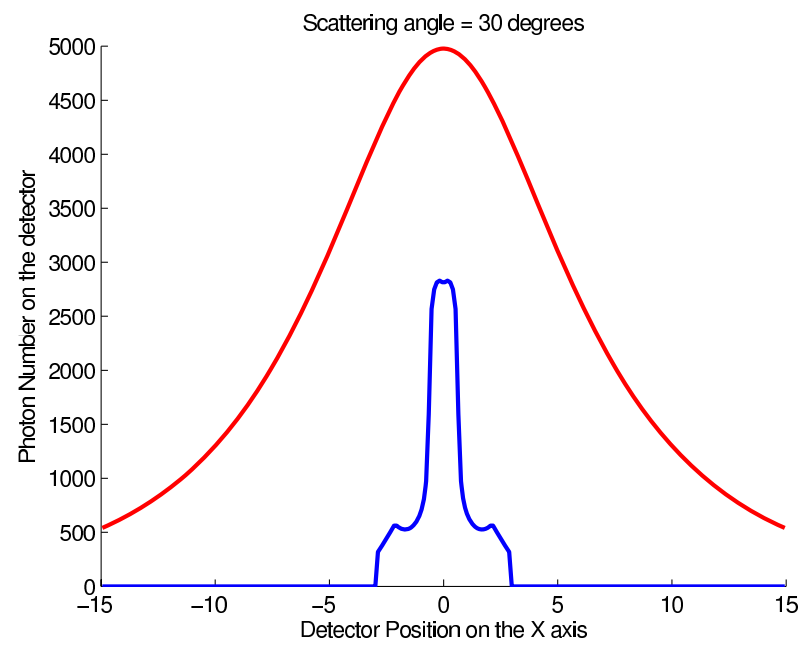

FIG. 8 Comparison of a typical PSF obtained using a parallel-hole (lower blue line) and using a slit collimator (upper red line).

the above described forward model were subsequently validated through extensive Monte Carlo simulations [17].

The 2D original object used as input to the simulation process (see Figure 9) is placed at the center of the scattering medium and a unit distance above the detector. A line detector of 55 pixels of 1 unit length, placed on the axis $y=0$, is simulated. We consider that the scattering medium has the same properties as soft tissue. It consists of a discretized square of $55 \times 55$ elements of unit area.

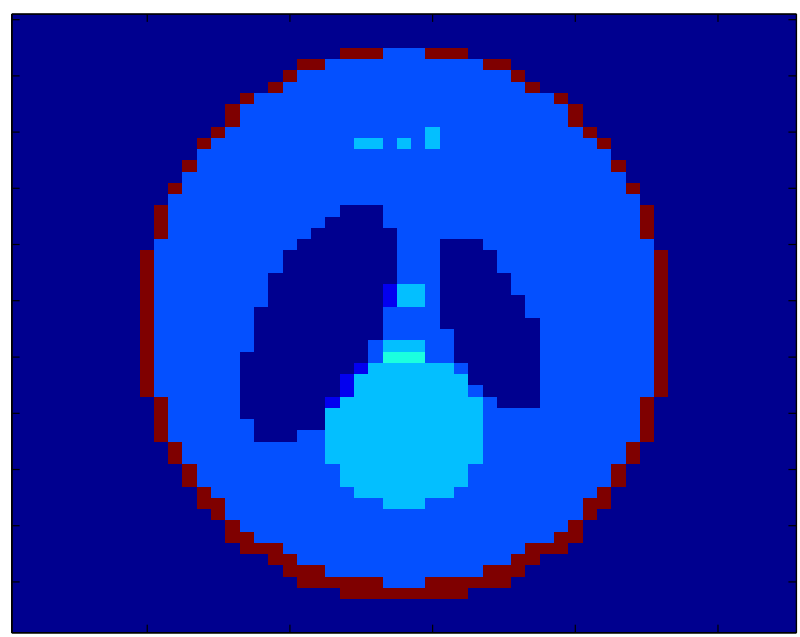

FIG. 9 Original Shepp-Logan phantom used as input to the simulation.

A series of 55 images of the object corresponding to 55 different scattering angles regularly distributed over the range $12^{\circ}<\omega<132^{\circ}$ (with a step of $2.18^{\circ}$ ) have been simulated. We construct the $3025 \times 3025$ weight matrix by computing, for each mesh point source, the PSF at the different scattering angles for each site on the detector. The reconstruction is carried out by inverting this weight matrix using the Singular Value Decomposition (SVD) method, which is less time consuming, compared to other reconstruction methods [18].

Figure 10 and Figure 11 show the reconstruction results ob- 
tained using both collimators. One can observe the better agreement with the original object when using the slit collimator. It can be seen in Figure 10 that the part of the object near the detector is better reconstructed than the upper part of the object where the three small structures are invisible. The reconstructed root mean square error (RMSE) is estimated to be about $9.13 \%$. However, when using the slit collimator (see Figure 11), the whole object is correctly reconstructed. All structures are visible and the RMSE is about $1.17 \times 10^{-4} \%$. These results have been subsequently validated by Monte Carlo simulations [17]. The objective assessment of image quality and quantitative accuracy achieved using the proposed approach is beyond the scope of this preliminary investigation and will be reported in future studies.

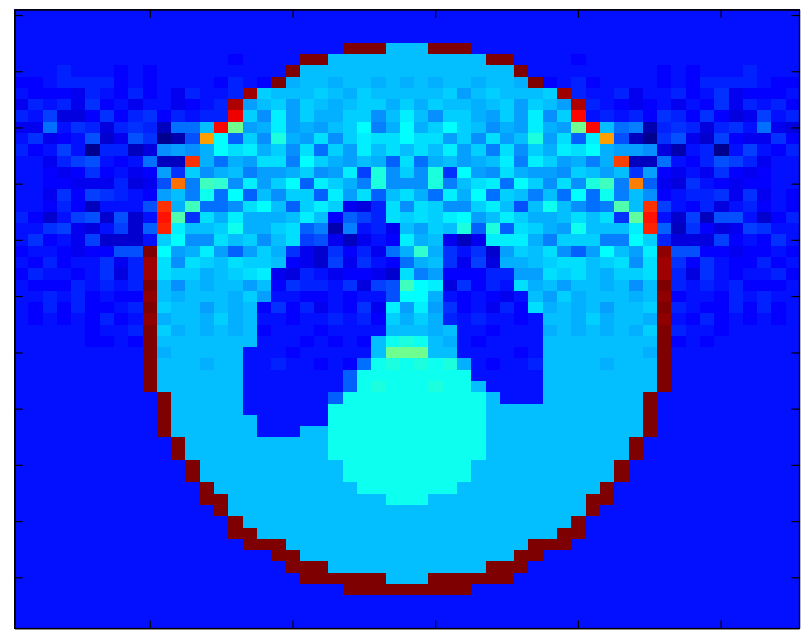

FIG. 10 Shepp-Logan phantom reconstruction corresponding to the geometry using a parallel-hole collimator.

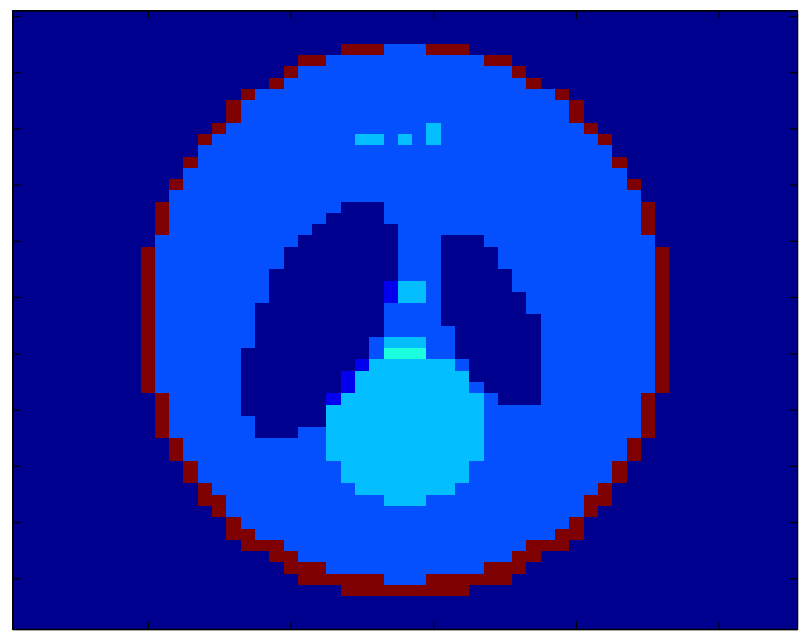

FIG. 11 Shepp-Logan phantom reconstruction corresponding to the geometry using a slit-collimator.

\section{CONCLUSION}

The feasibility of image reconstruction using Compton scattered photons detected by a gamma camera equipped with a slit collimator (in the two-dimensional case), operating in a stationary spatial position, is demonstrated in this study. This is the essence of a new concept of high sensitivity imaging, which takes advantage of scattered photons instead of rejecting them as done usually in conventional emission imaging. The main point in this imaging process by Compton scattered radiation is the fact that data acquisition is performed without the usual motion of the detector. This is a major advantage compared to existing imaging systems which require a heavy, bulky and costly mechanical rotation mechanism to move the detector around the patient in space. The motivation for presenting the concept in two-dimensions is motivated by the desire to keep the mathematical formulation as simple as possible, which can also be considered as an intermediate step and proof of concept towards the three-dimensional formulation. Work towards an extension to three-dimensional imaging using a more realistic non-uniform electron density map is in progress. The modelling and simulations of multiple scattered radiation in this context will be also subject of future investigation. These promising results may open the way to new high sensitivity imaging devices which might have applications in nuclear medicine imaging, non-destructive industrial control, high energy astrophysics, and environmental survey.

\section{ACKNOWLEDGEMENTS}

This work was supported by the Swiss National Science Foundation under grant No. 3152A0-102143 and by the French Ministry of Research under grant ACI NIM TRC.

\section{References}

[1] H. Zaidi and K. F. Koral, "Scatter modelling and compensation in emission tomography" Eur. J. Nucl. Med. Mol. I. 31, 761-782 (2004).

[2] M.K. Nguyen and T.T. Truong, "On an integral transform and its inverse in nuclear imaging" Inverse Probl. 18, 265-277 (2002).

[3] M.K. Nguyen and T.T. Truong and H.D. Bui and J.L. Delarbre, "A novel inverse problem in gamma-ray emission imaging" Inverse Probl. Sci. En. 12, 225-246 (2004).

[4] M.K. Nguyen and T. T. Truong and J. L. Delarbre and C. Roux and H. Zaidi, "Novel approach to stationary transmission scanning using Compton scattered radiation" Phys. Med. Biol. 52, 4615-4632, (2007).

[5] T. T. Truong and M. K. Nguyen and H. Zaidi, "The mathematical foundations of 3D Compton scatter emission imaging" International Journal of Biomedical Imaging doi: 10.1155/2007/92780 (2007).

[6] H. Zaidi, "Recent developments and future trends in nuclear medicine instrumentation" Med. Phys. 16, 5-17 (2006).

[7] R. L. Clarke and E. N. C. Milne and G. Van Dyk, "The use of Compton scattered gamma rays for tomography" Invest. Radiol. 11, 225-235 (1976).

[8] B. L. Evans and J. B. Martin and L. W. Burggaf and M. C. Roggemann. "Nondestructive Inspection Using Compton Scatter Tomography" IEEE T. Nucl. Sci. 45, no.3 (1998).

[9] E.M.A. Hussein, "Compton scatter imaging systems" in Bioinstrumentation: Research, Developments and Applications, Donald L. Wise, ed., chapter 35, 1053-1086 (Butterworths 1990).

[10] P.C. Johns and R.J. Leclair and M.P. Wismayer, "Medical X-ray Imaging with Scattered Photons" in Opto-Canada: SPIE Regional 
Meeting in Optoelectronics, Photonics and Imaging, SPIE TD 01, 355-357 (Ottawa, Canada 2002).

[11] S. J. Norton, "Compton scattering tomography" J. Appl. Phys. 76, 2007-2015 (1994).

[12] M. Singh, "An electronically collimated gamma camera for single photon emission computed tomography" Med. Phys. 10, 421-427 (1983).

[13] S. Chelikani and J Gore and G Zubal, “Optimizing Compton camera geometries" Phys. Med. Biol. 49, 1387-1408 (2004).

[14] L. Mihailescu and K. M. Vetter and M. T. Burks and E. L. Hull and W. W. Craig, "SPEIR: A Ge Compton camera" Nucl. Instrum. Meth. A 570, 89-100 (2007).
[15] G. L. Zeng and D. Gagnon, “CdZnTe strip detector SPECT imaging with a slit collimator" Phys. Med. Biol. 49, 2257-2271 (2004).

[16] H. Zaidi, "Relevance of accurate Monte Carlo modeling in nuclear medical imaging" Med. Phys. 26, 574-608 (1999).

[17] C. Driol and M.K. Nguyen and T.T. Truong, "Modelling and simulation results on high sensitivity scattered $\gamma$-ray imaging" in Proc. 6th EUROSIM Congress on Modelling and Simulation (LjubljanaSlovenia 2007).

[18] V. V. Selivanov and D. Lapointe and M. Bentourkia and R. Lecomte, "Cross validation stopping rule for ML-EM reconstruction of dynamic PET series effect" IEEE T. Nucl. Sci. 48, 883-889 (2001). 